\title{
Leonurine protects against ulcerative colitis by alleviating inflammation and modulating intestinal microflora in mouse models
}

\author{
SUNA ZHENG ${ }^{1}$, TIANCHI ZHUANG ${ }^{1}$, YAJUN TANG $^{1}$, RUIHAN WU $^{1}$, TING XU $^{1}$, \\ TIAN LENG ${ }^{1}$, YAO WANG ${ }^{2}$, ZHENG LIN $^{3}$ and MINGHUI JI ${ }^{1}$ \\ ${ }^{1}$ School of Nursing, Nanjing Medical University, Nanjing, Jiangsu 211166; ${ }^{2}$ Department of General Surgery, \\ The First Affiliated Hospital of Nanjing Medical University \& Jiangsu Province Hospital; \\ ${ }^{3}$ Department of Gastroenterology, The First Affiliated Hospital with Nanjing \\ Medical University, Nanjing, Jiangsu 210029, P.R. China
}

Received April 3, 2020; Accepted June 7, 2021

DOI: $10.3892 /$ etm.2021.10633

\begin{abstract}
Inflammatory bowel disease (IBD) is a chronic inflammatory disease of the colon. The aim of the present study was to explore the effects of leonurine (YMJ) on inflammation and intestinal microflora in colonic tissues of a dextran sulfate sodium (DSS)-induced ulcerative colitis (UC) mouse model. Mice were randomly divided into control $(n=5), \operatorname{DSS}(n=5$, treated with DSS) and DSS+YMJ ( $\mathrm{n}=5$, treated with DSS and YMJ) groups.Body weight was recorded, disease activity index (DAI) was calculated, and colon histopathology was evaluated using hematoxylin and eosin staining. Serum interleukin (IL)-6, tumor necrosis factor- $\alpha$ (TNF- $\alpha)$ and IL- $1 \beta$ levels were examined using ELISA. Expression levels of nuclear factor- $\kappa \mathrm{B}$ (p65) and phosphorylated (p)-p65 were evaluated via western blotting. 16S ribosomal RNA was extracted from mouse feces. Composition or abundance changes of intestinal microflora were analyzed. The results indicated that YMJ treatment (DSS+YMJ group) significantly increased body weight, reduced DAI scores and increased colon length in UC mouse models compared with those in the DSS group $(\mathrm{P}<0.05)$. YMJ significantly reduced inflammatory infiltration, significantly decreased serum TNF- $\alpha$, IL- 6 and IL-1 $\beta$ levels $(\mathrm{P}<0.05)$ and significantly downregulated the $\mathrm{p}-\mathrm{p} 65 / \mathrm{p} 65$ ratio compared with the DSS
\end{abstract}

Correspondence to: Dr Minghui Ji, School of Nursing, Nanjing Medical University, 101 Longmian Avenue, Nanjing, Jiangsu 211166, P.R. China

E-mail: jiminghui@njmu.edu.cn

Dr Yao Wang, Department of General Surgery, The First Affiliated Hospital of Nanjing Medical University \& Jiangsu Province Hospital, 300 Guangzhou Road, Gulou, Nanjing, Jiangsu 210029, P.R. China

E-mail: wangyao198904@163.com

Key words: inflammatory bowel disease, leonurine, ulcerative colitis, intestinal microflora group $(\mathrm{P}<0.05)$. YMJ increased the quantity of the intestinal flora and improved intestinal microflora diversity in the mice of the DSS group. Specifically, YMJ partly regulated intestinal microflora in feces, including a reduction of Bifidobacterium, and an increase in Parasutterella and Ackermania. In conclusion, YMJ improved disease outcomes of the UC mice, reduced the levels of serum inflammatory factors and increased the ratio of beneficial bacteria in the intestinal tract.

\section{Introduction}

Inflammatory bowel disease (IBD), which primarily manifests as ulcerative colitis (UC) or Crohn's disease, is considered a chronic inflammatory disease of the rectum and colon with an unknown etiology $(1,2)$. IBD is mainly characterized by abdominal pain, diarrhea, mucus, purulent stool, and pathological lesions limited to the mucosa and sub-mucosa $(1,2)$. The prevalence of IBD is higher in Europe and USA; however, the morbidity of IBD in Asian countries is increasing yearly, particularly in China, which has the highest rate amongst Asian countries. At present, the morbidity of UC in China is $~ 3.44$ per 100,000 individuals (3). The pathogenic process of UC is associated with dysregulation of the intestinal flora and intestinal mucosal immune disarrangements (4), and is characterized by bacterial diversity loss and transfer of microflora $(5,6)$. It has been demonstrated that the dextran sulfate sodium (DSS)-induced colitis model is the best UC model for simulating the pathological processes of UC, including abnormal cytokine production, and infiltration of neutrophils and macrophages into colonic epithelial cells $(7,8)$. In a previous study on DSS-induced mouse models, the number of lactic acid bacteria decreased notably, whereas that of Vibrio desulfuricans significantly increased, suggesting that such changes in the microflora maybe closely associated with intestinal inflammation (9). Numerous studies on the intestinal microflora have been performed to prevent or treat UC (10), including transplantation of fecal bacteria, which achieves its therapeutic effect by returning the normal functionality of the intestinal flora (11). Clinically, numerous immunomodulators 
and non-steroidal anti-inflammatory drugs (such as sulfadiazine and glucocorticoids) have been widely applied for treating UC $(12,13)$. However, the above therapeutic strategies usually cause various adverse effects, such as vomiting, hepatorenal toxicity, systemic edema and anemia $(10,11)$. Traditional Chinese Medicines (TCMs) possess strong anti-inflammatory effects with fewer side-effects (14). As a result, TCMs have attracted increasing attention due to their anti-inflammatory properties, particularly as a treatment for IBD (15-17).

Leonurine (YMJ), as an active ingredient, was first extracted as a TCM from Leonurus heterophyllus (18). YMJ promotes uterine contraction, and prevents osteoporosis, mastitis and myocardial fibrosis (19). The above functions of YMJ maybe associated with its anti-inflammatory effects. Previous studies reported that YMJ could inhibit neuro-inflammation, suppress the $\mathrm{PI} 3 \mathrm{~K} / \mathrm{AKT} / \mathrm{NF}-\kappa \mathrm{B}$ signaling pathway and prevent endometritis (20-22). Therefore, it was speculated that YMJ may also possess beneficial effects as a treatment for UC. TCMs prevent and treat IBD by regulating the intestinal microflora, such as Chlorogenic acid, Escherichia coli, F. prausnitzii and Clostridium pratense (23). The effects of YMJ on UC and the associated mechanisms have not been explored, to the best of our knowledge. Therefore, the present study evaluated the preventative effects of YMJ on IBD, and whether they involved changes in the intestinal flora.

The aim of the present study was to investigate the therapeutic effects of YMJ on DSS-induced UC in C57BL/6J mice, and to explore the functional regulation of $\mathrm{YMJ}$ on intestinal flora in mice. Improvement of colitis in mice was evaluated by estimating changes in weight, disease activity score and colon histopathology. In order to examine the effects of YMJ on intestinal inflammation in IBD, the cytokine levels in serum were determined by ELISA, whereas the infiltration of immune cells in the colonic tissues of mice were evaluated using immunohistochemical analysis. The mechanism of YMJ-mediated regulation of intestinal flora in mice was evaluated using 16S ribosomal RNA (rDNA) sequencing, and by estimating the changes in the abundance and composition of fecal microflora.

\section{Materials and methods}

Animals. C57BL/6J wild-type mice (weighting 18-20 g; 8 weeks old) were purchased form Shanghai SLAC Laboratory Animal Co., Ltd. The mice were maintained in standard cages in a specific pathogen free animal room at a temperature of $22 \pm 2^{\circ} \mathrm{C}$ and a humidity of $55 \pm 5 \%$, with a light/dark cycle of $12 \mathrm{~h}$. All mice were provided ad libitum access to sterilized water and standard chow. This study was performed in accordance with Guide of the Care and Use of Laboratory Animals published by the US National Institutes of Health (NIH) (24). The present study was approved by the Ethical Committee of Nanjing Medical University (Nanjing, China) (approval no. IACUC-1904036).

Trial grouping, UC model establishment and sample collection. A total of 15 mice were randomly divided into three groups: A control group $(n=5)$, a DSS group $(n=5)$ and a DSS+YMJ group $(n=5)$. During the 15 days of experiments, the mice were administered different treatments. The mice in the control group were provided double-distilled water for 15 days ad libitum, without any other treatments. In the DSS group, the mice were provided double-distilled water for 7 days ad libitum, followed by double-distilled water containing 2.5\% DSS (cat. no. 42867; Sigma-Aldrich; Merck KGaA) for the subsequent 8 days. In the DSS+YMJ group, the mice were provided double-distilled water containing $1 \mathrm{mM}$ YMJ (cat. no. PA12123; Weng Jiang Reagent Co., Ltd.) for 7 days, followed by administration of double-distilled water containing $2.5 \%$ DSS for the 8 subsequent days.

Following the above treatments for 15 days, the skin surrounding the anus was disinfected with $75 \%$ ethanol to stimulate defecation. Then, the feces of mice were collected and stored in a sterile Eppendorf tube at $-80^{\circ} \mathrm{C}$ for extracting DNA and bacterial microflora. The mice were monitored closely daily for signs of pain, distress and behavior, through evaluating the appetite, activity levels and hydration status. During the above processes, no mice died in any of the groups. The mice were euthanized using $150 \mathrm{mg} / \mathrm{kg}$ pentobarbital (Sigma-Aldrich; Merck KGaA), and a $0.1 \mathrm{ml}$ retro-orbital blood sample was collected(centrifuged at room temperature for $2 \mathrm{~h}$ at 2,000 $\mathrm{x}$ g)for detection of inflammatory factors in the serum, and the whole colonic tissues of mice were obtained and fixed in neutral formalin solution for $2 \mathrm{~h}$ at room temperature for subsequent experiments. The death of the mice was confirmed by cardiac arrest, respiratory arrest, pupillary dilation and the absence of a nerve reflex.

Evaluation of weight and disease activity. During the experiment, the weight of the mice was recorded at the same time every day, and the weight-change curve was drawn accordingly. Mice exhibiting blood in their stool and moribund mice were also observed. The severity of DSS-induced colitis was evaluated using the disease activity index (DAI), which involves three assessing indices, as illustrated in Table I (25). The DAI value in this study was defined as the average score of the above three assessing indices. Mice were anesthetized by intraperitoneal injection of pentobarbital $(50 \mathrm{mg} / \mathrm{kg}$; Sigma-Aldrich; Merck KGaA) and then were euthanized using $150 \mathrm{mg} / \mathrm{kg}$ pentobarbital. The entire large intestine was separated from the anal to the ileocecal junction, and the length of the colon between the ileocecal junction and the anus was measured.

Hematoxylin and eosin (H\&E) staining. Colon histopathology was evaluated by $H \& E$ staining. Briefly, $24 \mathrm{~h}$ after fixing with neutral formalin, the mice colonic tissues were dehydrated using a graded series of alcohol solutions and embedded in paraffin. The embedded colonic tissues were sliced into sections of a thickness of $5 \mu \mathrm{m}$. The sections were placed in an oven and heated at $65^{\circ} \mathrm{C}$ for $30 \mathrm{~min}$. Next, the sections were stained with hematoxylin (cat. no. C0107; Beyotime Institute of Biotechnology) and eosin (cat. no. C0109; Beyotime Institute of Biotechnology), as reported elsewhere (26). Finally, the sections were sealed with neutral resin and scanned using a light microscope (Ax70; Olympus Corporation) (magnification, $\mathrm{x} 400$ ) with CellSens Standard v.1.6 software (Olympus Corporation).

ELISA. The expression of inflammatory factors in serum was determined using ELISA. Briefly, retro-orbital blood $(0.1 \mathrm{ml})$ 
Table I. Scoring system for disease activity index.

\begin{tabular}{lcll}
\hline Score & Weight loss & Fecal consistency & \multicolumn{1}{c}{ Bleeding } \\
\hline 0 & 0 & Normal & No \\
1 & $0-5 \%$ & Slight changed & Trace \\
2 & $5-10 \%$ & Mild diarrhea & Mild occult blood \\
3 & $10-20 \%$ & Diarrhea & Significant bleeding \\
4 & $>20 \%$ & Severe diarrhea & Massive bleeding \\
\hline
\end{tabular}

was collected from mice and incubated for $2 \mathrm{~h}$ at room temperature, and then centrifuged at $2,000 \mathrm{xg}$ at $4^{\circ} \mathrm{C}$ for $10 \mathrm{~min}$. Next, the serum levels of interleukin (IL)-6, tumor necrosis factor- $\alpha$ $($ TNF- $\alpha$ ) and IL-1 $\beta$ were detected using a Mouse IL-6 ELISA kit (cat. no. PI326), Mouse TNF- $\alpha$ ELISA kit (cat. no. PT512) and Mouse IL-1 $\beta$ ELISA kit (cat. no. PI301), respectively (all from Beyotime Institute of Biotechnology).

Western blot analysis. The expression levels of inflammatory proteins, including NF- $\mathrm{KB}$ (also known as p65) and phosphorylated (p)-p65, in mouse colonic tissues were evaluated by western blotting. The colonic tissues were cut into small pieces and treated with nucleoprotein lysate $(200 \mu \mathrm{l})$ (cat. no. P0013; Beyotime Institute of Biotechnology) to obtain protein lysates. The concentration of protein lysates was determined using a BCA Protein assay kit (cat. no. P0010S, Beyotime Institute of Biotechnology). A total of $0.5 \mu \mathrm{g}$ protein (per lane) lysates were then subjected to SDS-PAGE, and then electro-transferred to PVDF membranes (Amersham; Cytiva). The PVDF membranes were first blocked with 5\% bovine serum albumin (cat. no. A8806; Sigma-Aldrich; Merck KGaA) at room temperature for $1 \mathrm{~h}$, and then incubated with a rabbit anti-mouse p65 polyclonal antibody (cat. no. ab16502; 1:2,000), rabbit anti-mouse p65 (phospho S316, phosphorylated p65, p-p65) polyclonal antibody (cat. no. ab254097; 1:2,000) and rabbit anti-mouse GAPDH monoclonal antibody (cat. no. ab181602; $1: 2,000$ ) at $4^{\circ} \mathrm{C}$ overnight. Subsequently, the PVDF membranes were incubated with horseradish peroxidase-labeled goat anti-rabbit IgG antibody (cat. no. ab205718; 1:1,000) at room temperature for $2 \mathrm{~h}$. All of the above antibodies were purchased from Abcam. Finally, signals were visualized using BeyoECL Moon Imaging kit (cat. no. P0018FS; Beyotime Institute of Biotechnology). The western blotting images were captured using Quantity One version 4.6.0 (Bio-Rad Laboratories, Inc.).

Extraction of $16 S$ rDNA from mouse feces, and biological analysis of composition and abundance changes in intestinal microflora. DNA was extracted from mouse feces using a DNA extraction kit (cat. no. D0065S; Beyotime Institute of Biotechnology), according to the manufacturer's instructions. Briefly, a 200-mg feces sample was added to a 2-ml centrifugal tube and subjected to a high centrifugation speed of $10,000 \mathrm{x} \mathrm{g}$ at $4^{\circ} \mathrm{C}$ for $45 \mathrm{~min}$. Next, the supernatants were discarded, and the precipitates were re-suspended using $100 \mathrm{ml}$ pre-cooled PBS and treated with phenol, followed by centrifugation of the solutions at a speed of $10,000 \mathrm{xg}$ at $4^{\circ} \mathrm{C}$ for $10 \mathrm{~min}$. Finally, the purified genomic DNA was extracted by adding pre-cooled absolute ethanol. The concentration of total DNA was detected using an ultraviolet spectrophotometer (NanoDrop 2000 UV; NanoDrop Technologies; Thermo Fisher Scientific, Inc.). The 16S rDNA extraction, biological analysis and abundance changes in intestinal microflora were performed using the Meiji cloud platform as described in previous studies $(27,28)$. The above genomic DNA was used to generate the 16S rDNA. The V3, V4 and V5 hypervariable regions of the prokaryotic 16S rDNA were used for synthesizing the amplicons. The DNA libraries were validated using the Agilent Bioanalyzer (Agilent Technologies, Inc.) and quantified with a Qubit 2.0 fluorometer. Then, the DNA libraries were analyzed using the Illumina MiSeq platform (Illumina, Inc.) and MiSeq Control Software v.2.2.0 (Illumina, Inc.). The raw reads were filtered and matched to the sequences spanning the V3-V4 amplicon with PANDAseq, as described previously (29). The merged sequences with $97 \%$ identity of nucleotide sequence were clustered into operational taxonomic units (OTUs) using UPARSE (30). The abundance, diversity, similarity and composition of the bacteria were generated as described previously (28). Moreover, the principal co-ordinates analysis was also conducted according to the previously studies reported (28-30).

Statistical analysis. Continuous variables were represented as the mean \pm standard deviation of at least 6 repeats, and analyzed using SPSS (version 20.0; IBM Corp.). ANOVA followed by a Tukey's post-hoc test was used to compare differences between multiple groups. $\mathrm{P}<0.05$ was considered to indicate a statistically significant difference.

\section{Results}

YMJ enhances body weight and reduces the DAI score in the UC mice. The weight of the mice in the control group increased steadily, and the mice did not exhibit diarrhea or hematochezia. At $\sim 4$ days post-DSS induction (DSS group), diarrhea and blood in the stool were recorded, and the weight of the mice significantly decreased compared with that of the control mice (Fig. 1A; $\mathrm{P}<0.05$ ). However, the diarrhea and blood in the stool of mice in the TMJ-treated group (DSS+YMJ group) was notably reduced, and the weight of mice in DSS+YMJ group was significantly higher compared with that of mice in the DSS group from days $12-15$ (Fig. $1 \mathrm{~A} ; \mathrm{P}<0.05$ ). Meanwhile, the weight of mice in DSS+YMJ group was also significantly lower compared with that of mice in the control group from days 12-15 (Fig. 1A; P<0.05). Furthermore, the DAI values were significantly lower in the DSS+YMJ group compared with the DSS group from days 12-15 (Fig. 1B; $\mathrm{P}<0.05$ ). These results suggested that the YMJ could improve the body weight via reducing the DAI scores in UC mice, hence, the reasons for how YMJ triggered body weight enhancement was investigated in the following experiments.

$Y M J$ treatments increases the length of the colon in the UC mice. To determine the effect of YMJ on the colon length of the UC mice, the length of the colons of the treated mice was measured. The results showed that, compared with the control group, the colon length of mice in the DSS group was significantly shortened (Fig. 1C and D; $\mathrm{P}<0.05$ ).However, the length 

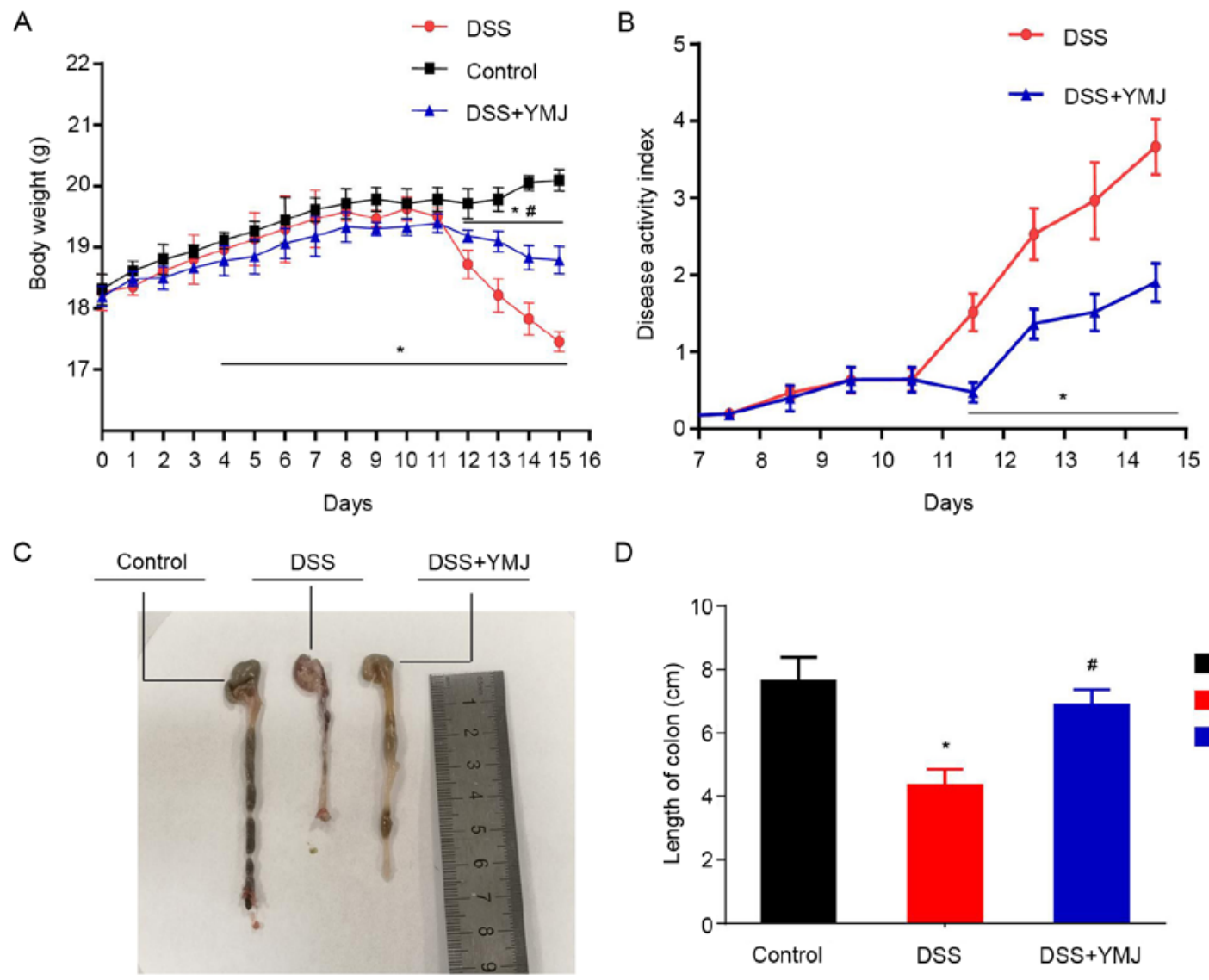

D

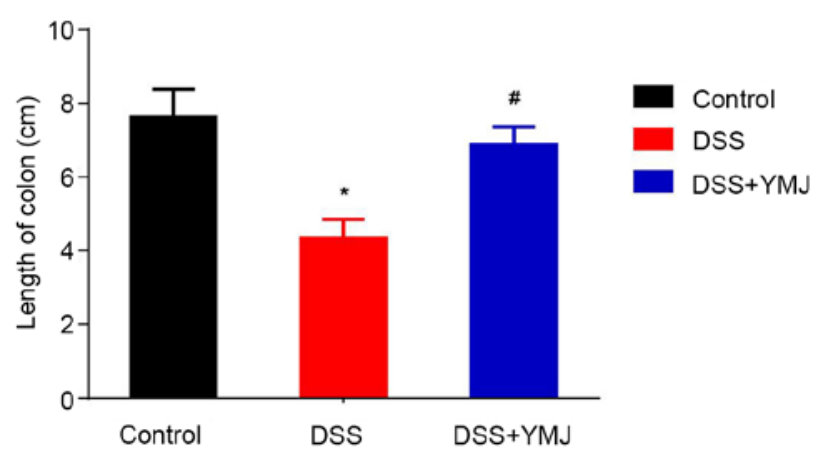

Figure 1. Effects of YMJ treatment on the body weight, DAI scores and colon length of mice in different groups. (A) Effects of YMJ on the body weight of mice. (B) Effects of YMJ on DAI scores. (C) Comparison of colon length across all groups. (D) Statistical analysis of colon length amongst the three groups ${ }^{\text {}} \mathrm{P}<0.05$ vs. control group; ${ }^{*} \mathrm{P}<0.05$ vs. DSS group. DAI, disease activity index. YMJ, leonurine; DSS, dextran sulfate sodium.

of the colon in the YMJ-treated mice (DSS+YMJ group) was significantly lengthened compared with that in the DSS mice (Fig. $1 \mathrm{C}$ and $\mathrm{D} ; \mathrm{P}<0.05$ ). These results suggest that $\mathrm{YMJ}$ significantly alleviates the UC induced by DSS.

YMJ alleviates inflammatory infiltration. The H\&E staining findings indicated that the mucosal epithelium of mice in the blank control group was intact and the glands were well arranged, without congestion, edema, ulcers or inflammatory cell infiltration (Fig. 2A). The cell atypia of the DSS group was obvious, and it was accompanied by extensive inflammatory cell infiltration (Fig. 2B). The intestinal mucosa of mice in the DSS+YMJ group was clear at all levels, and inflammatory cell infiltration could be observed locally, but cell atypia was not obvious (Fig. 2C). Furthermore, in the DSS group, the histological structure of the colon was lost, including disintegration of the epithelium, damage of the barrier and a decrease of the crypt, which was accompanied by infiltration of granulocytes and single nuclear cells into the mucosa and sub-mucosa (Fig. 2B). By contrast, in the YMJ-treated mice, the extent and severity of colon injury were significantly reduced (Fig. 2C).

$Y M J$ decreases the serum levels of inflammatory factors in the UC mice. The serum levels of inflammatory factors in the UC mice were examined by ELISA. The results showed that the serum levels of TNF- $\alpha$ (Fig. 3A), IL-6 (Fig. 3B) and IL-1 $\beta$ (Fig. 3C) were significantly higher in the mice of the
DSS group compared with that of the mice in the control group $(\mathrm{P}<0.05)$. The $\mathrm{YMJ}$ treatment significantly decreased the serum levels of inflammatory factors compared with that observed in the mice of the DSS group (Fig. 3A; $\mathrm{P}<0.05$ ). However, the serum levels of TNF- $\alpha$ (Fig.3A), IL-6 (Fig. 3B) and IL-1 $\beta$ (Fig. 3C) in the DSS+YMJ group were also significantly lower than that in the control group $(\mathrm{P}<0.05)$.

$Y M J$ downregulates the $N F-\kappa B$ signaling pathway in the UC mice. The western blotting results (Fig. 4A) demonstrated that compared with the control group, the p-p65/p65 ratio was significantly higher in the DSS group (Fig. 4B; $\mathrm{P}<0.01$ ). Notably, the p-p65/p65 ratio was significantly downregulated in the DSS+YMJ group compared with that in the DSS group (Fig. 4B; $\mathrm{P}<0.05$ ). Therefore, DSS activated the NF- $\kappa \mathrm{B}$ signaling pathway, whereas YMJ inhibited this pathway.

YMJ increases the quantity of intestinal flora in mice. The fecal DNA content of each sample was $>100 \mathrm{ng}$, and the optical density 260/280 ratio was 1.8-2.0, which met the amplification requirements (Fig. 5A). After adding $>25,000$ sequences, only a small number of OTUs were produced, suggesting that the sequencing quantity (number of base reads) was appropriate. The analysis of the similarity of intestinal microflora using Venn diagrams showed that there were more intestinal microflora in the DSS+YMJ group than in the control and DSS groups (Fig. 5B; $\mathrm{P}<0.05$ ). However, there were no significant 
Table II. Top 10 species at the genus levels in feces of mice.

\begin{tabular}{|c|c|c|c|c|c|}
\hline \multirow[b]{2}{*}{$\begin{array}{l}\text { Control } \\
\text { Genus }\end{array}$} & \multirow[b]{2}{*}{$\begin{array}{l}\text { Constituent } \\
\text { ratio }\end{array}$} & \multicolumn{2}{|l|}{ DSS } & \multicolumn{2}{|l|}{$\mathrm{DSS}+\mathrm{YMJ}$} \\
\hline & & Genus & $\begin{array}{l}\text { Constituent } \\
\text { ratio }\end{array}$ & Genus & $\begin{array}{l}\text { Constituent } \\
\text { ratio }\end{array}$ \\
\hline Bifidobacterium & $67.52 \%$ & Escherichia coli-Shigella & $15.25 \%$ & Parasutterella & $30.80 \%$ \\
\hline Ruminococcus & $5.11 \%$ & Helicobacter & $14.65 \%$ & Ackermania & $10.17 \%$ \\
\hline Polymorphic bacillus & $4.75 \%$ & Bifidobacterium & $13.72 \%$ & Polymorphic bacillus & $12.05 \%$ \\
\hline Lachnospira & $3.90 \%$ & Turicibacter & $12.58 \%$ & Escherichia coli-Shigella & $11.76 \%$ \\
\hline Lactobacillus & $1.83 \%$ & Polymorphic bacillus & $11.88 \%$ & Turicibacter & $4.84 \%$ \\
\hline Ackermania & $1.73 \%$ & Mucispirillum & $5.60 \%$ & Parabacterioides & $4.42 \%$ \\
\hline Streptococcus & $1.33 \%$ & Enterococcus & $3.00 \%$ & Helicobacter & $4.00 \%$ \\
\hline \multicolumn{6}{|l|}{ fragmentosus } \\
\hline Rikenellaceae & $1.30 \%$ & Non-classified streptococcus & $2.69 \%$ & Bifidobacterium & $3.73 \%$ \\
\hline Paraprentium & $1.02 \%$ & Parasutterella & $2.10 \%$ & Lactobacillus & $2.25 \%$ \\
\hline Parabacterioides & $0.92 \%$ & Ackermania & $2.03 \%$ & Lachnospira & $1.72 \%$ \\
\hline
\end{tabular}

DSS, dextran sulfate sodium; YMJ, leonurine.

A

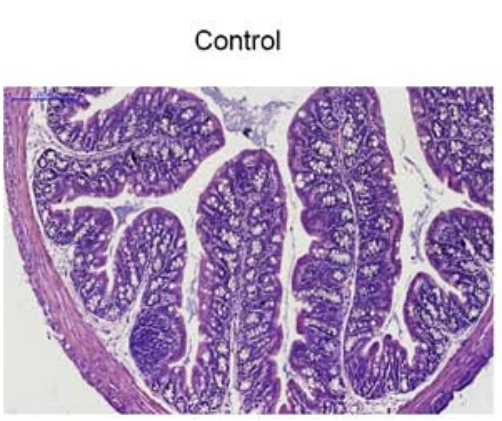

B

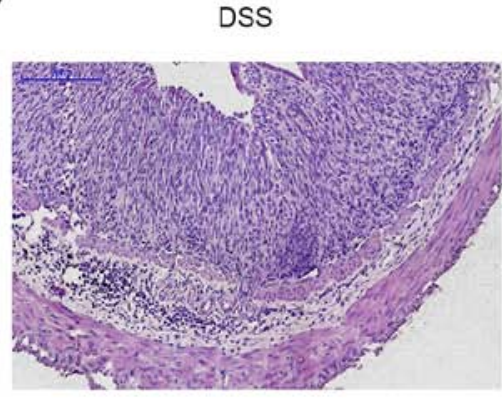

C

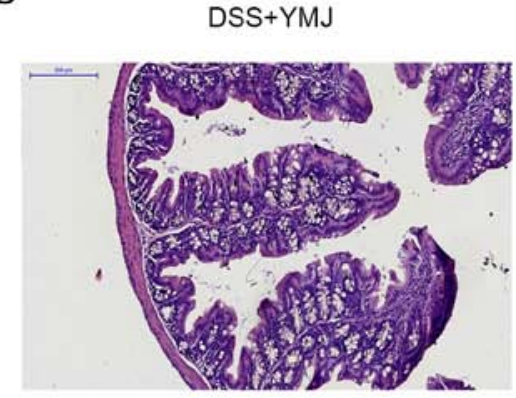

Figure 2. H\&E staining images. H\&E images of inflammatory cell infiltration and severity of colon injury in the (A) control, (B) DSS and (C) DSS+YMJ groups. YMJ, leonurine; DSS, dextran sulfate sodium; H\&E, hematoxylin and eosin.

differences in the quantity of intestinal microflora between the control and DSS groups (Fig. 5B; $\mathrm{P}>0.05$ ).

YMJ improves intestinal microflora diversity in DSS mice. To further visualize similarities or differences amongst the DSS+YMJ group and mice in the other two groups, principal co-ordinates analysis was performed based on weighted UniFrac distance (Fig. 6). In the intra-group analysis, the sample distances in each group were close, and the differences were small; thus, they could be clustered into groups. The poor degree of aggregation of samples in the DSS group indicated that the changes to the bacterial flora caused by DSS were irregular. Regarding the distance between groups, the DSS+YMJ group was closer to the control group than it was to the DSS group. However, the difference between mice in the YMJ-treated group and the DSS group was not significant, suggesting that YMJ treatment could improve the intestinal flora diversity of DSS mice slightly.

Top intestinal microflora at the phylum level in the feces of mice. The results of intestinal flora structure analysis showed that the top six species in each group were Bacteroidetes, Firmicutes, Proteobacteria, Verrucomicrobia,Tenericutes and
Deferribacteres. The dominant bacteria in each group were the same, namely Bacteroidetes, Firmicutes, Proteobacteria and Verrucomicrobia (Fig. 7) Therefore, compared with those of the control group, the order and composition ratio of dominant bacteria at the phylum level in the DSS group exhibited a substantial change, and Bacteroidetes decreased significantly, whereas Firmicutes, Proteobacteria, Verrucomicrobia, Tenericutes and Deferribacteres increased significantly.

Compared with the DSS group, the order of microflora level in the DSS+YMJ group was consistent, although the composition ratio was slightly different (Fig. 7). The differences were primarily observed in Tenericutes $(0.42 \%$ in the DSS+YMJ group, $1.91 \%$ in the DSS group and $0.55 \%$ in the control group) and Deferribacteres (0.14\% in the DSS+YMJ group, $5.55 \%$ in the DSS group and $0.08 \%$ in the control group). The order of microflora level and the composition ratio in the DSS+YMJ group were close to those of the control group.

Top 10 intestinal microflora at the genus level in the feces of mice. The present study further specified annotations at the genus level and listed the top 10 intestinal microflora, as shown in Table II. The results revealed that the highest proportion of 
A

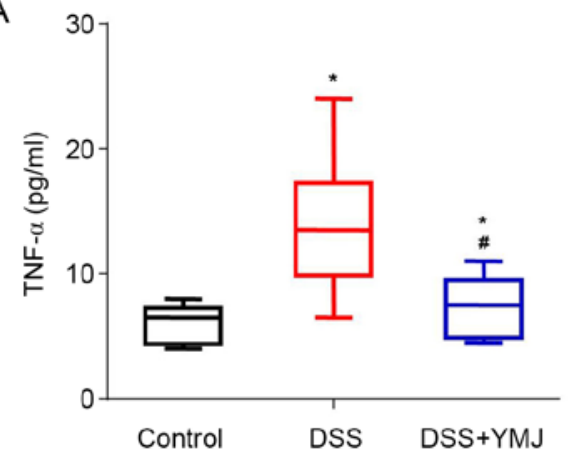

C

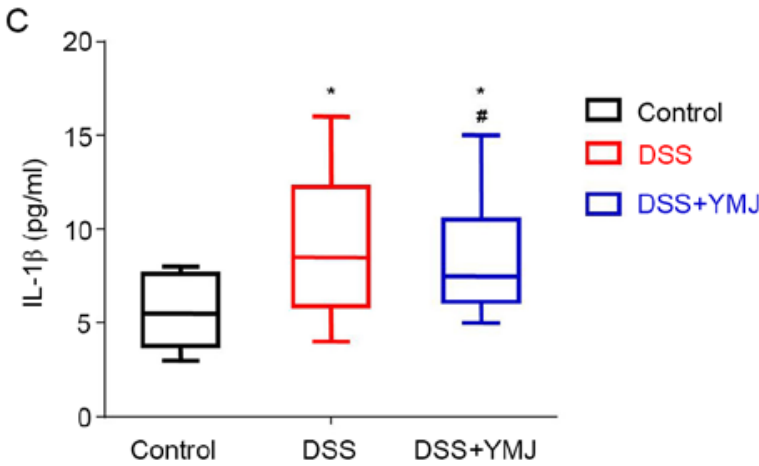

B

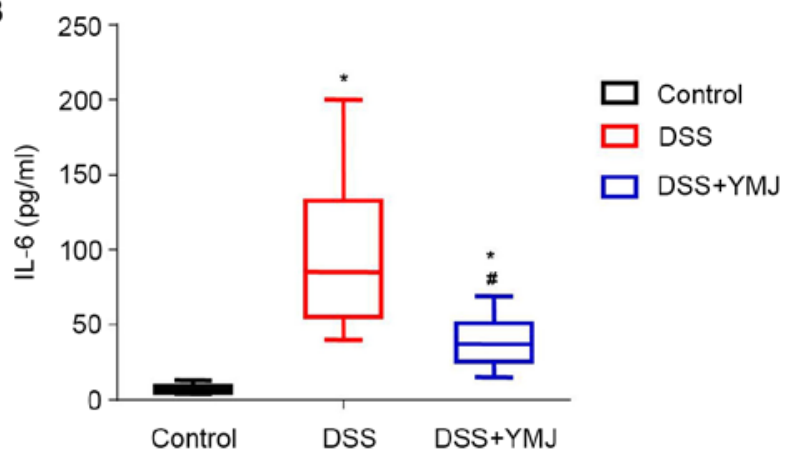

Figure 3. Evaluation of serum levels of inflammatory factors in the mice of each group using ELISA. Statistical analysis of the serum levels of (A) TNF- $\alpha$, (B) IL-6 and (C) IL-1ßin the mice of each group. ${ }^{*} \mathrm{P}<0.05$ vs. control group; ${ }^{*} \mathrm{P}<0.05$ vs. DSS group. IL, interleukin; YMJ, leonurine; DSS, dextran sulfate sodium; TNF- $\alpha$, tumor necrosis factor- $\alpha$.

A

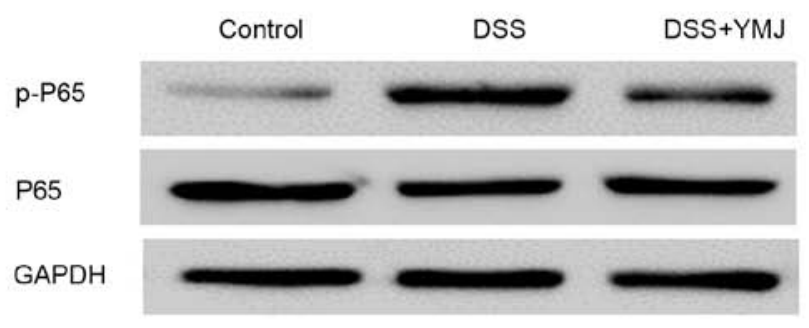

B

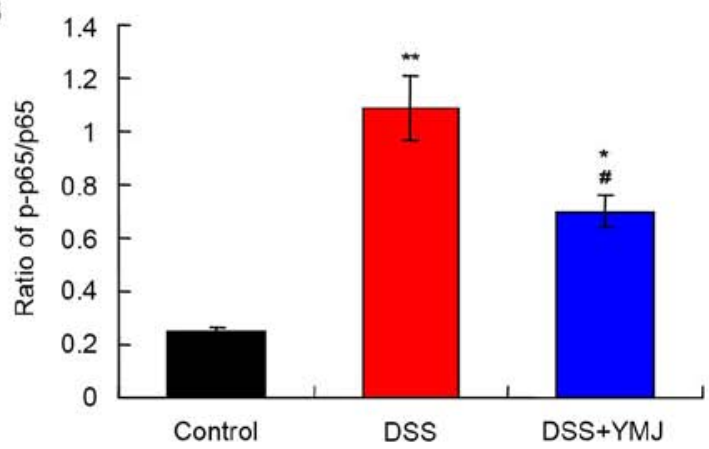

Figure 4. Determination of p65 and p-p65 expression in the mice of each group using western blot analysis. (A) Western blotting images of p65 and p-p65. (B) Statistical analysis of p65 and p-p65 expression. ${ }^{*} \mathrm{P}<0.05,{ }^{* *} \mathrm{P}<0.01$ vs. control group; ${ }^{*} \mathrm{P}<0.05$ vs. DSS group. p-, phosphorylated; YMJ, leonurine; DSS, dextran sulfate sodium.

Escherichia coli-Shigella was found in the DSS group (15.25\%), followed by Helicobacter (14.65\%), Bifidobacterium (13.72\%), Turicibacter (12.58\%), Polymorphic bacillus (11.88\%) and Mucispirillum (5.60\%). After treatment with YMJ, the dominant microflora in the intestinal tract of mice were probiotics, including Parasutterella (30.80\%), Ackermania (10.17\%), Polymorphic bacillus (12.05\%), Escherichia coli-Shigella $(11.76 \%)$, Turicibacter $(4.84 \%)$ and Parabacterioides $(4.42 \%)$. The intestinal microflora in the mice of the control group primarily included Bifidobacterium (67.52\%), Ruminococcus (5.11\%) and Polymorphic bacillus (4.75\%).

\section{Discussion}

The present study explored the effects of YMJ on DSS-induced mouse models of UC. The DSS-induced UC mouse model is a common experimental method used to investigate intestinal inflammation (31). Previous studies have shown that YMJ can improve mucosal injury, and can reduce the extent and severity of injury $(22,32)$. In addition, YMJ could also alleviate the inflammatory damage induced by DSS through inhibition of the NF- $\kappa \mathrm{B}$ signaling pathway and decreasing the secretion of inflammatory factors (33). Furthermore, YMJ can inhibit pathogenic bacteria, such as Escherichia coli-Shigella, increase the proportion of Parasutterella and Ackermania, and alleviate intestinal inflammation (33).

Intestinal mucosa, as a mucosal layer of the intestinal wall, prevents the entrance of toxins and pathogens into the intestinal cavity (34). Once the mucosal barrier is penetrated, the sub-mucosal layer will be exposed to a large number of intraluminal antigens, including food, bacteria and cytokines that are produced in the process of the innate immune response. 
A

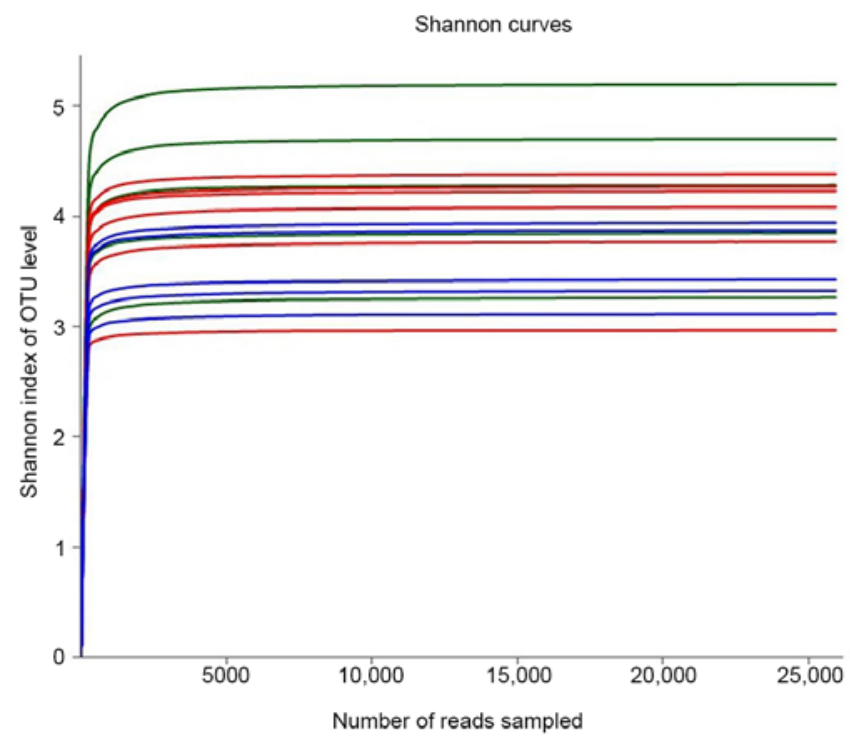

B
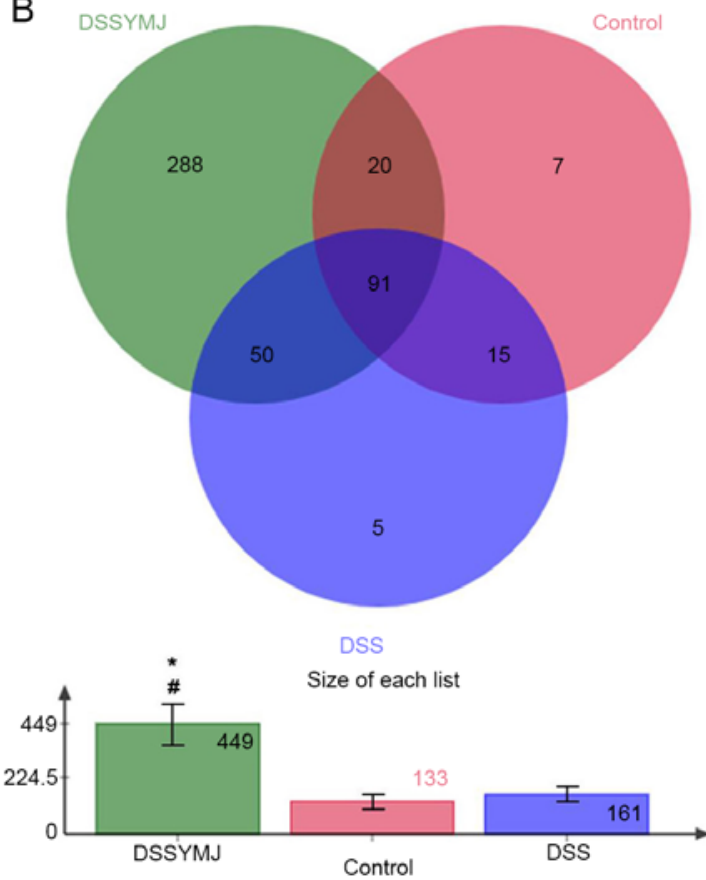

Number of elements: specific (1) or shared by $2,3, \ldots$ lists

\begin{tabular}{c|c|c|}
\hline 91 & 85 & 300 \\
\hline 3 & 2 & 1
\end{tabular}

Figure 5. Effects of leonurine on the levels of intestinal microflora in the mice of each group. (A) Dilution curve of each group. (B) Similarity Venn diagram of intestinal microflora in the mice of each group. The numbers in the Venn diagram represent the OTUs. ${ }^{*} \mathrm{P}<0.05$ vs. Control; ${ }^{*} \mathrm{P}<0.05$ vs. DSS group. YMJ, leonurine; DSS, dextran sulfate sodium; OTU, operational taxonomic unit.

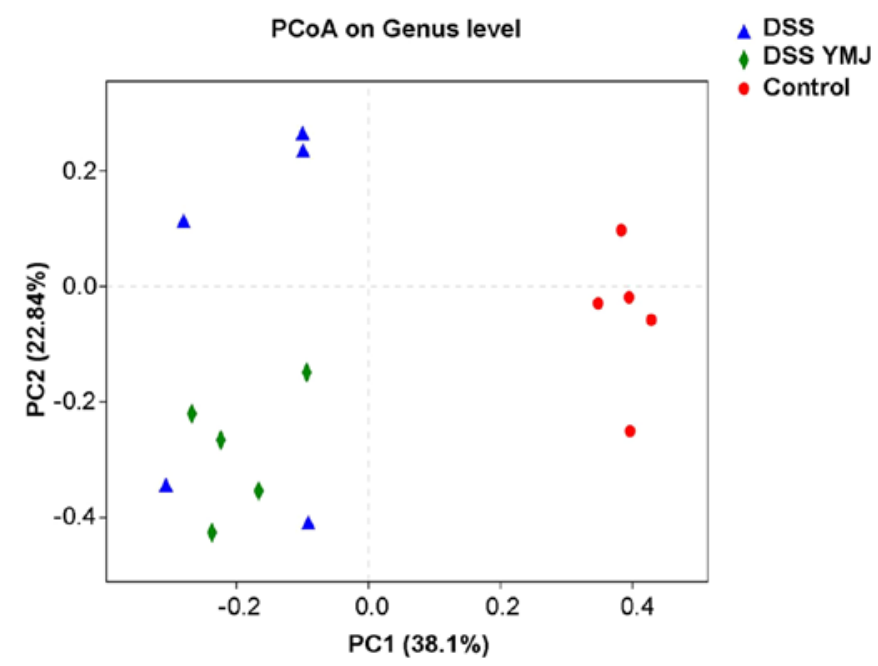

Figure 6. PCoA of the intestinal microflora diversity in the mice of each group. PCoA, Principal co-ordinates analysis; PC, principal co-ordinate; DSS, dextran sulfate sodium; TCM, traditional Chinese medicine.

Histopathological evaluation showed that YMJ could alleviate mucosal injury, improve weight loss and the DAI score of mice, and alleviate diarrhea and hematochezia (35).

YMJ inhibited the $N F-\kappa B$ signaling pathway, which resulted in reduced infiltration of immune cells and a decrease in the secretion of inflammatory cytokines (33). When NF- $\mathrm{BB}$ is not activated, it forms a complex with $\mathrm{I} \kappa \mathrm{B} \alpha$. When inflammatory factors, growth factors or chemokines (such as TNF- $\alpha$ and IL-1 $\beta$ ) in the colon of mice activate $N F-\kappa B, I \kappa B \alpha$ is phosphorylated and then degraded by the ubiquitin-proteasome signaling pathway (36). TNF- $\alpha$, IL- 6 and IL- $1 \beta$ have also been demonstrated to act as proinflammatory factors, which are important for modulating inflammation. The results of the present study showed that YMJ decreased the serum levels of inflammatory factors, including TNF- $\alpha$, IL- 6 and IL- $1 \beta$, in the UC mouse model. Furthermore, YMJ modulated the NF- $\kappa \mathrm{B}$ signaling pathway by downregulating the p-p65/p65 ratio. These results suggest that DSS activates the NF- $\mathrm{BB}$ signaling pathway, and YMJ notably inhibited the activation of the $\mathrm{NF}-\kappa \mathrm{B}$ signaling pathway toa certain extent, and reduced the inflammatory damage in the UC mouse model.

Bacteroidetes and Firmicutes are the two most abundant phyla in the human intestinal tract, followed by Proteobacteria, Verrucomicrobia and Verrucomicrobia. The composition of intestinal microflora in the C57BL/6J wild-type mice is similar to that of the human intestinal flora (37); thus, mice were selected as animal models in the present study. At the UC stage, changes in intestinal microflora always trigger associated inflammatory pathways to further expand the inflammatory response, which leads to the deterioration of the disease $(38,39)$. The present findings indicated that YMJ could improve the abundance and distribution of bacteria; therefore, the anti-inflammatory effects of YMJ may be closely associated with the regulation of intestinal microflora. YMJ could reverse the dominant position of two types of inflammatory bacteria in the intestinal tract of model mice, such as Parasutterella and Ackermania. In addition, YMJ could increase the abundance and proportion of probiotics in the intestinal tract of experimental mice, including Bifidobacterium, Bacillus, Lactococcus 
A

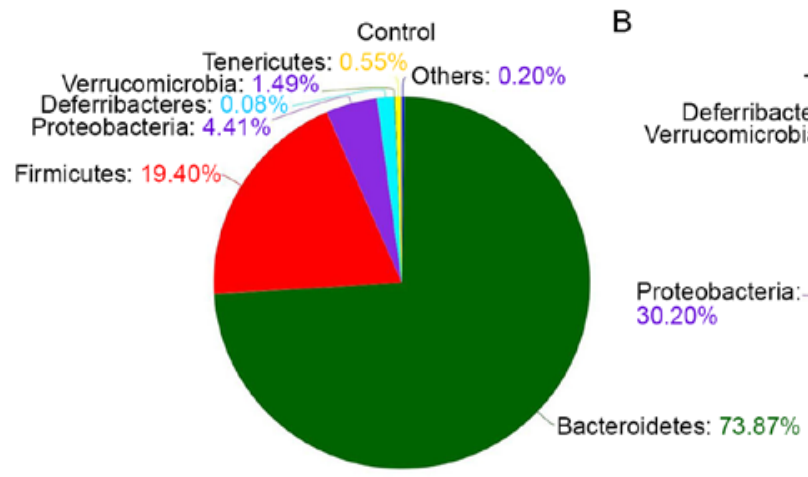

C

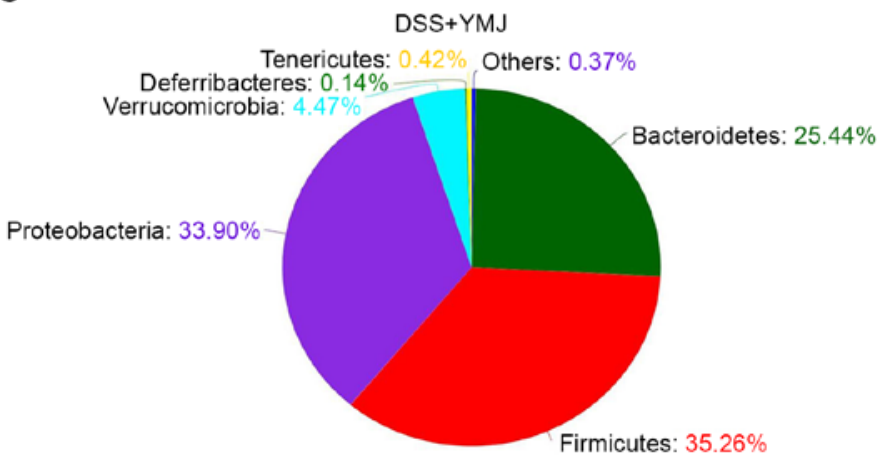

DSS

Tenericutes: $1.91 \%$ Others: $0.37 \%$

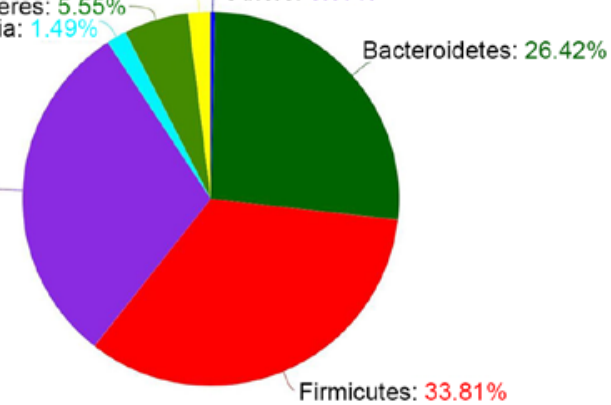

Figure 7. Graphs representing the top six intestinal microflora at the phylum level in the feces of mice. Top six intestinal microflora at the phylum level in the (A) control, (B) DSS and (C) DSS+YMJ groups. DSS, dextran sulfate sodium group. YMJ, leonurine; DSS, dextran sulfate sodium.

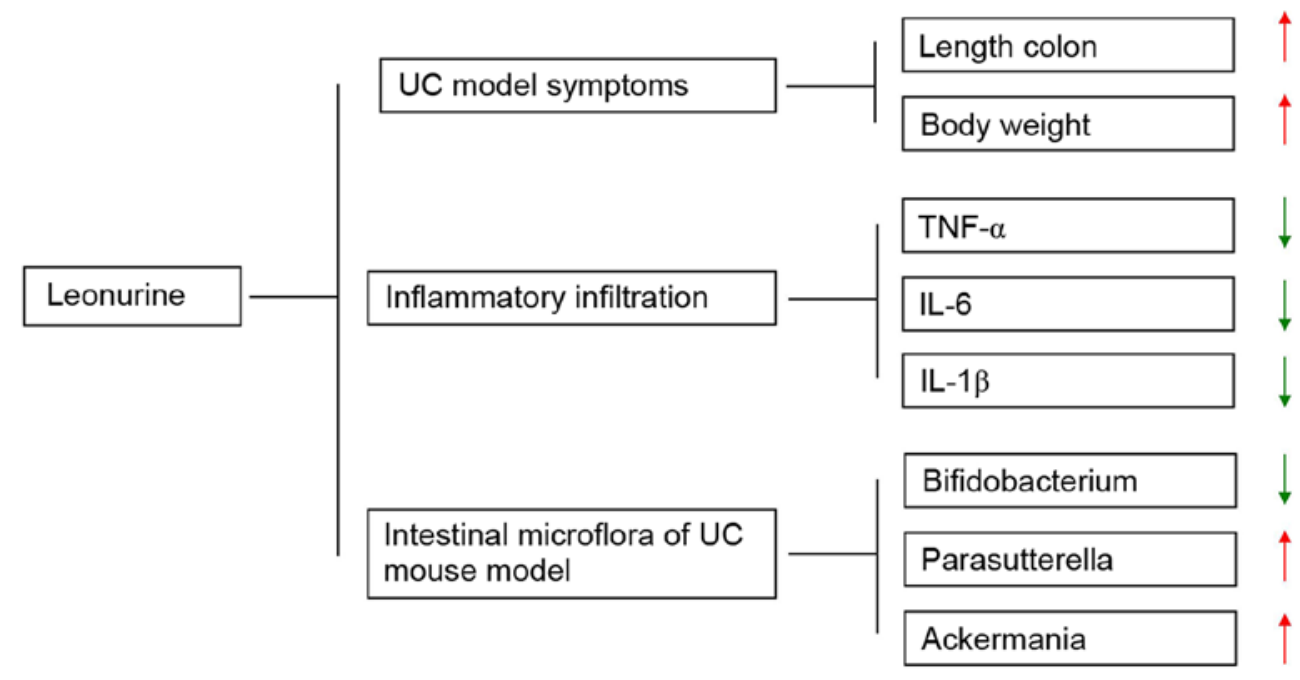

Figure 8. Pathway illustration of the association between leonurine, inflammatory response, microflora and UC models. The red arrows represent upregulation, and the green arrows represent downregulation. UC, ulcerative colitis; TNF- $\alpha$, tumor necrosis factor- $\alpha$; IL, interleukin.

and Lactobacillus. Following YMJ treatment, the levels of Parasutterella, which belongs to the core symbiotic bacteria, increased significantly. Parasutterella can stably colonize the intestinal tract of mice without initiating an immune response or inducing fluctuations of the intestinal flora (40). A previous study reported that bacteria maybe involved in the maintenance of bile acid homeostasis and cholesterol metabolism through alterations in bile acid transporter genes in the ileum, and bile acid synthesis genes in the liver (41). YMJ also induced the increase in Ackermania, a bacterium that degrades mucoprotein in the intestines. Notably, Ackermania is negatively correlated with obesity, diabetes, inflammation and metabolic disorders $(42,43)$, and positively correlated with behavioral indicators of anxiety and depression (44-46). Ackermania can regulate intestinal mucus thickness and maintain intestinal integrity $(46,47)$. The present study demonstrated that YMJ could reduce inflammatory factors to prevent the initiation of inflammation, inhibit pathogenic bacteria, such as Escherichia coli and Shigella, and increase the proportion of Parasutterella and Ackermania. Therefore, YMJ could further reduce intestinal inflammation, improve intestinal microflora and alleviate the severity of UC. Furthermore, the species and abundance of intestinal microflora in mice undergoing YMJ treatment were improved. 
The present study has various limitations. First, a positive drug group was not included, since this is a preliminary study on the effect of YMJ on DSS-induced UC. In subsequent studies, the effect of YMJ should be investigated and compared with positive drugs. Second, the correlation between YMJ and intestinal microflora also requires further study, which would be beneficial for clinical application. Third, the targeting bacteria screened in this study have not been studied in depth. Further research on YMJ treatment for UC is required. Finally, due to the preliminary experimental findings on the safety of YMJ, the effects of leonurine treatment on the microflora of normal mice have not been investigated. In future studies, the effects of YMJ should be determined.

In conclusion, YMJ improved the disease outcomes of UC to a certain extent, reduced the serum levels of inflammatory factors and increased the ratio of beneficial bacteria in the intestinal tract (Fig. 8). Therefore, YMJ has clinical potential and value for the treatment of UC through modulating the intestinal microflora and reducing the inflammatory response in the intestinal tract. Future studies should focus on the selected targeting of intestinal microflora, and clarify the correlation between YMJ and intestinal microflora in UC animal models.

\section{Acknowledgements}

Not applicable.

\section{Funding}

This work was funded by the Natural Science Foundation of Jiangsu province (grant no. BK20180678), the Natural Science Foundation of Jiangsu Universities (grant no. 17KJB330004), Project of 'Nursing Science' Funded by the Priority Academic Program Development of Jiangsu Higher Education Institutions (General Office, the People's Government of Jiangsu Province; grant no. [2018]-No. 87), the key discipline project of 'Nursing Science' of Jiangsu province during the 13th five-year plan (Jiangsu Provincial Primary and Secondary School Teaching and Research Office; grant no. [2016]-No. 9), 'Nursing Science' of Jiangsu University Brand Professional Construction Project (Jiangsu Colleges and Universities; grant no. [2015]-No. 11) and 'Centre for Health Promotion and Nursing Cooperative Innovation' Constructive Project of Nanjing Medical University (grant no. JX21831803/004).

\section{Availability of data and materials}

The datasets used and/or analyzed during the present study are available from the corresponding author on reasonable request. The sequencing data generated during this study using samples obtained from experimental animals have been upload to China National GeneBank database (db.cngb.org/; accession no. CNP0001816).

\section{Authors' contributions}

YW, ZL, MJ conceived and designed the experiments. SZ, TZ, YT, RW and TX performed experiments and analyzed the data. SZ and TZ contributed to analysis. YW wrote the manuscript and performed the statistical analysis. TL performed the experiments and literature review. SZ, YW, ZL, MJ confirmed the authenticity of all the raw data. All authors read and approved the final manuscript.

\section{Ethics approval and consent to participate}

The present study was performed in accordance with the Guide of Care and Use of Laboratory Animals of National Institutes of Health (revised in 1996). The present study was approved by the Ethical Committee of Nanjing Medical University (Nanjing, China) (approval no. IACUC-1904036).

\section{Patient consent for publication}

Not applicable.

\section{Competing interests}

The authors declare that they have no competing interests.

\section{References}

1. Yu YR and Rodriguez JR: Clinical presentation of Crohn's, ulcerative colitis, and indeterminate colitis: Symptoms, extraintestinal manifestations, and disease phenotypes. Semin Pediatr Surg 26: 349-355, 2017.

2. Cheng C, Hua J, Tan J, Qian W, Zhang L and Hou X: Identification of differentially expressed genes, associated functional terms pathways, and candidate diagnostic biomarkers in inflammatory bowel diseases by bioinformatics analysis. Exp Ther Med 18: 278-288, 2019.

3. Ng SC, Tang W, Ching JY, Wong M, Chow CM, Hui AJ, Wong TC, Leung VK, Tsang SW, Yu HH, et al; Asia-Pacific Crohn's and Colitis Epidemiologic Study (ACCESS) Study Group: Incidence and phenotype of inflammatory bowel disease based on results from the Asia-pacific Crohn's and colitis epidemiology study. Gastroenterology 145: 158-165.e2, 2013.

4. Sartor RB and Wu GD: Roles for intestinal bactetria, viruses, and fungi in pathogenesis of inflammatory bowel diseases and therapeutic approaches. Gastroenterology 152: 327-339.e4, 2017.

5. Kostic AD, Xavier RJ and Gevers D: The microbiome in inflammatory bowel disease: Current status and the future ahead. Gastroenterology 146: 1489-1499, 2014.

6. Michail S, Durbin M, Turner D, Griffiths AM, Mack DR, Hyams J, Leleiko N, Kenche H, Stolfi A and Wine E: Alterations in the gut microbiome of children with severe ulcerative colitis. Inflamm Bowel Dis 18: 1799-1808, 2012.

7. Chen ZM, Peto R, Iona A, Guo Y, Chen YP, Bian Z, Yang L, Zhang YY, Lu F, Chen JS, Collins R, Li LM and China Kadoorie Biobank Collaborative Group: Emerging tobacco-related cancer risks in China: A nationwide, prospective study of 0.5 million adults. Cancer 121 (Suppl 17): 3097-106, 2915.

8. Lin Y, Yang X, Yue W, Xu X, Li B, Zou L and He R: Chemerin aggravates DSS-induced colitis by suppressing M2 macrophage polarization. Cell Mol Immunol 11: 355-366, 2014.

9. Håkansson Å, Tormo-Badia N, Baridi A, Xu J, Molin G, Hagslätt ML, Karlsson C, Jeppsson B, Cilio CM and Ahrné S: Immunological alteration and changes of gut microbiota after dextran sulfate sodium (DSS) administration in mice. Clin Exp Med 15: 107-120, 2015.

10. Paramsothy S, Kamm MA, Kaakoush NO, Walsh AJ, van den Bogaerde J, Samuel D, Leong RW, Connor S, Ng W, Paramsothy R, Xuan W, Lin E, Mitchell HM and Borody TJ: Multidonor intensive faecal microbiota transplantation for active ulcerative colitis: a randomised placebo-controlled trial. Lancet 389: 1218-28, 2017.

11. Weingarden AR and Vaughn BP: Intestinal microbiota, fecal microbiota transplantation, and inflammatory bowel disease. Gut Microbes 8: 238-252, 2017.

12. Ishiguro Y, Ohkawara T, Sakuraba H, Yamagata K, Hiraga H, Yamaguchi S, Fukuda S, Munakata A, Nakane A and Nishihira J: Macrophage migration inhibitory factor has a proinflammatory activity via the p38 pathway in glucocorticoid-resistant ulcerative colitis. Clin Immunol 120: 335-341, 2006. 
13. Zhou H, Wong YF, Wang J, Cai X and Liu L: Sinomenine ameliorates arthritis via MMPs, TIMPs, and cytokines in rats. Biochem Biophys Res Commun 376: 352-357, 2008.

14. Yu XH, Wang YF, Dai FY, Zhao JH and Li P: The protective effects of Berberine and Hesperidin on inflammatory factor-stimulating cardiac fibroblasts. Eur Rev Med Pharmacol Sci 23 5468-5476, 2019.

15. Li W, Zhao X, Lv X, Han W and Wang H: Silibinin retards colitis-associated carcinogenesis by repression of $\mathrm{Cdc} 25 \mathrm{C}$ in mouse model. Inflamm Bowel Dis 25: 1187-1195, 2019.

16. DeVore NM and Scott EE: Nicotine and 4-(methylnitrosamino) -1-(3-pyridyl)-1-butanone binding and access channel in human cytochrome P450 2A6 and 2A13 enzymes. J Biol Chem 287: 26576-26585, 2012.

17. Zhu L, Gu P and Shen H: Protective effects of berberine hydrochloride on DSS-induced ulcerative colitis in rats. Int Immunopharmacol 68: 242-251, 2019.

18. Liang H, Liu P, Wang Y, Song S and Ji A: Protective effects of alkaloid extract from Leonurus heterophyllus on cerebral ischemia reperfusion injury by middle cerebral ischemic injury (MCAO) in rats. Phytomedicine 18: 811-818, 2011.

19. Yang D, Jia W and Zhu YZ: Leonurine, a potential agent of traditional Chinese medicine: Recent updates and future perspectives. Nat Prod Commun 11: 1757-1761, 2016.

20. Jin M, Li Q, Gu Y, Wan B, Huang J, Xu X, Huang R and Zhang Y: Leonurine suppresses neuroinflammation through promoting oligodendrocyte maturation. J Cell Mol Med 23: 1470-1485, 2019

21. Hu ZC, Gong LF, Li XB, Fu X, Xuan JW, Feng ZH and Ni WF: Inhibition of PI3K/Akt/NF- $\kappa \mathrm{B}$ signaling with leonurine for ameliorating the progression of osteoarthritis: In vitro and in vivo studies. J Cell Physiol 234: 6940-6950, 2019.

22. Wu H,Dai A, Chen X, Yang X,Li X, Huang C, Jiang K and Deng G: Leonurine ameliorates the inflammatory responses in lipopolysaccharide-induced endometritis. Int Immunopharmacol 61 $156-161,2018$.

23. Zhang Z, Wu X, Cao S, Cromie M, Shen Y, Feng Y, Yang H and Li L: Chlorogenic acid ameliorates experimental colitis by promoting growth of Akkermansia in mice. Nutrients 9: E677, 2017.

24. US National Institutes of Health (NIH): Guide of the Care and Use of Laboratory Animals. NIH Publication, pp852-853, 1996.

25. Han F, Zhang H, Xia X, Xiong H, Song D, Zong X and Wang Y: Porcine $\beta$-defensin 2 attenuates inflammation and mucosal lesions in dextran sodium sulfate-induced colitis. J Immunol 194 1882-1893, 2015

26. Liang YN, Yu JG, Zhang DB, Zhang Z, Ren LL, Li LH, Wang Z and Tang ZS: Indigo Naturalis ameliorates dextran sulfate sodium-induced colitis in mice by modulating the intestinal microbiota community. Molecules 24: 4086, 2019.

27. Zeng SL, Li SZ, Xiao PT, Cai YY, Chu C, Chen BZ, Li P, Li J and Liu EH: Citrus polymethoxyflavones attenuate metabolic syndrome by regulating gut microbiome and amino acid metabolism. Sci Adv 6: eaax6208, 2020.

28. Ye Z, Zhang N, Wu C, Zhang X, Wang Q, Huang X, Du L, Cao Q, Tang J, Zhou C, et al: A metagenomic study of the gut microbiome in Behcet's disease. Microbiome 6: 135, 2018.

29. Masella AP, Bartram AK, Truszkowski JM, Brown DG and Neufeld JD: PADNAseq: Paired-end assembler for illumina sequences. BMC Bioinformatics 13: 1-7, 2013.

30. Edgar RC: UPARSE: Highly accurate OTU sequences from microbial amplicon reads. Nat Methods 10: 996-998, 2013.

31. Zhang WF, Yang Y, Su X, Xu DY, Yan YL, Gao Q and Duan MH: Deoxyschizandrin suppresses dss-induced ulcerative colitis in mice. Saudi J Gastroenterol 22: 448-455, 2016.

32. Nie J and Liu X: Leonurine attenuates hyperalgesia in mice with induced adenomyosis. Med Sci Monit 23: 1701-1706, 2017.

33. Li N, Xu Q, Liu Q, Pan D, Jiang Y, Liu M, Liu M, Xu H and Lin C: Leonurine attenuates fibroblast-like synoviocyte-mediated synovial inflammation and joint destruction in rheumatoid arthritis. Rheumatology (Oxford) 56: 1417-1427, 2017.
34. Olson A, Diebel LN and Liberati DM: Exogenous phosphatidylcholine supplementation improves intestinal barrier defense against Clostridium difficile toxin. J Trauma Acute Care Surg 77: 570-575, discussion 576, 2014.

35. Shang X, Pan H, Wang X, He H and Li M: Leonurus japonicus Houtt.: Ethnopharmacology, phytochemistry and pharmacology of an important traditional Chinese medicine. J Ethnopharmacol 152: 14-32, 2014.

36. Shen J, Cheng J, Zhu S, Zhao J, Ye Q, Xu Y, Dong H and Zheng X: Regulating effect of baicalin on $\mathrm{IKK} / \mathrm{IKB} / \mathrm{NF}-\kappa \mathrm{B}$ signaling pathway and apoptosis-related proteins in rats with ulcerative colitis. Int Immunopharmacol 73: 193-200, 2019.

37. Haange SB, Jehmlich N, Hoffmann M, Weber K, Lehmann J, von Bergen $\mathrm{M}$ and Slanina U: Disease development is accompanied by changes in bacterial protein abundance and functions in a refined model of dextran sulfate sodium (DSS)-induced colitis. J Proteome Res 18: 1774-1786, 2019.

38. Rodríguez-Nogales A, Algieri F, Garrido-Mesa J, Vezza T, Utrilla MP, Chueca N, Fernández-Caballero JA, García F, Rodríguez-Cabezas ME and Gálvez J: The administration of Escherichia coli Nissle 1917 ameliorates development of DSS-induced colitis in mice. Front Pharmacol 9: 468, 2018.

39. Li M, Wu Y, Hu Y, Zhao L and Zhang C: Initial gut microbiota structure affects sensitivity to DSS-induced colitis in a mouse model. Sci China Life Sci 61: 762-769, 2018.

40. Chen YJ, Wu H, Wu SD, Lu N, Wang YT, Liu HN, Dong L, Liu TT and Shen XZ: Parasutterella, in association with irritable bowel syndrome and intestinal chronic inflammation. J Gastroenterol Hepatol 33: 1844-1852, 2018.

41. Ju T, Kong JY, Stothard P and Willing BP: Defining the role of Parasutterella, a previously uncharacterized member of the core gut microbiota. ISME J 13: 1520-1534, 2019.

42. Sheng L, Jena PK, Liu HX, Hu Y, Nagar N, Bronner DN, Settles ML, Bäumler AJ and Wan YY: Obesity treatment by epigallocatechin-3-gallate-regulated bile acid signaling and its enriched Akkermansia muciniphila. FASEB J: Jun 8, 2018 (Epub ahead of print). doi: 10.1096/fj.201800370R.

43. Moreira GV, Azevedo FF, Ribeiro LM, Santos A, Guadagnini D, Gama P, Liberti EA, Saad M and Carvalho C: Liraglutide modulates gut microbiota and reduces NAFLD in obese mice. J Nutr Biochem 62: 143-154, 2018.

44. Demirci M, Tokman HB, Uysal HK, Demiryas $S$, Karakullukcu A, Saribas S, Cokugras H and Kocazeybek BS: Reduced Akkermansia muciniphila and Faecalibacterium prausnitzii levels in the gut microbiota of children with allergic asthma. Allergol Immunopathol (Madr) 47: 365-371, 2019.

45. Kosciow K and Deppenmeier U: Characterization of a phospholipid-regulated beta-galactosidase from Akkermansia muciniphila involved in mucin degradation. Microbiologyope: Feb6, 2019.

46. McGaughey KD, Yilmaz-Swenson T, Elsayed NM, Cruz DA, Rodriguiz RM, Kritzer MD, Peterchev AV, Roach J, Wetsel WC and Williamson DE: Relative abundance of Akkermansia spp. and other bacterial phylotypes correlates with anxiety- and depressive-like behavior following social defeat in mice. Sci Rep 9: 3281, 2019.

47. Cruz-Aguliar RM, Wantia N, Clavel T, Vehreschild MJ, Buch T, Bajbouj M, Haller D, Busch D, Shmid RM and SteinThoeringer CK: An open-labeled study on fecal microbiota transfer in irritable bowel syndrome patients reveals improvement in abdominal pain associated with the relative abundance of Akkermansia muciniphila. Digestion 100: 127-138, 2019.

This work is licensed under a Creative Commons Attribution-NonCommercial-NoDerivatives 4.0 International (CC BY-NC-ND 4.0) License. 